\title{
Correction: Worldwide Network for Blood and Marrow \\ Transplantation (WBMT) perspective: the role of biosimilars in hematopoietic cell transplant: current opportunities and challenges in low- and lower-middle income countries
}

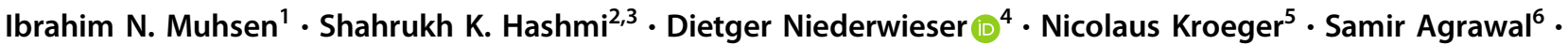 \\ Marcelo C. Pasquini ${ }^{7} \cdot$ Yoshiko Atsuta $^{8} \cdot$ Karen K. Ballen $^{9} \cdot$ Adriana Seber $^{10} \cdot$ Wael Saber $^{7}$. \\ Mohamed A. Kharfan-Dabaja $\mathbb{1}^{11} \cdot$ Walid Rasheed $^{2} \cdot$ Shinichiro Okamoto ${ }^{12} \cdot$ Nandita Khera $^{13} \cdot$ William A. Wood $^{14}$. \\ Mickey B. C. Koh ${ }^{15} \cdot$ Hildegard Greinix $^{16} \cdot$ Yoshihisa Kodera $^{17} \cdot$ Jeff Szer $\mathbb{D}^{18} \cdot$ Mary M. Horowitz ${ }^{7}$. Daniel Weisdorf ${ }^{19}$. \\ Mahmoud Aljurf ${ }^{2}$
}

Published online: 15 October 2019

(c) The Author(s), under exclusive licence to Springer Nature Limited 2019

\section{Correction to: Bone Marrow Transplant} https://doi.org/10.1038/s41409-019-0658-2
Following publication, it was noted that an authors name was misspelt. Dietger Niederweiser has been corrected to Dietger Niederwieser.
Shahrukh K. Hashmi

hashmi.shahrukh@mayo.edu

1 Department of Medicine, Houston Methodist Hospital, Houston, TX, USA

2 Hematology Department, King Faisal Specialist Hospital \& Research Centre, Riyadh, Saudi Arabia

3 Department of Medicine, Mayo Clinic, Rochester, MN, USA

4 Department of Hematology and Medical Oncology, University of Leipzig, Leipzig, Germany

5 Department of Stem Cell Transplantation, University Hospital Hamburg-Eppendorf, Hamburg, Germany

6 Division of Haemato-Oncology, Bart's Health NHS Trust and Blizard Institute, Queen Mary University of London, London, UK

7 Center for International Blood and Marrow Transplant Research (CIBMTR), Milwaukee, WI, USA

8 Japanese Data Center for Hematopoietic Cell Transplantation, Nagoya, Japan

9 Division of Hematology/Oncology, University of Virginia School of Medicine, Charlottesville, VA, USA
10 Hospital Samaritano de, Sao Paulo, Brazil

11 Department of Medicine, Division of Hematology-Oncology and Blood and Marrow Transplantation program, Mayo Clinic, Jacksonville, FL, USA

12 Keio University School of Medicine, Tokyo, Japan

13 Department of Medicine, Mayo Clinic Arizona, Phoenix, AZ, USA

14 Lineberger Comprehensive Cancer Center, University of North Carolina-Chapel Hill, Chapel Hill, NC, USA

15 St George University Hospital, London, United Kingdom

16 Medical University of Graz, LKH-University, Klinikum Graz, Austria

17 Center for Hematopoietic Stem Cell Transplantation, Aichi Medical University Hospital, Nagakute, Japan

18 Department of Clinical Haematology, Royal Melbourne Hospital, Melbourne, VIC, Australia

19 University of Minnesota, Minneapolis, MN, USA 